\title{
Females in the forefront: time-based intervention effects on impulsive choice and interval timing in female rats
}

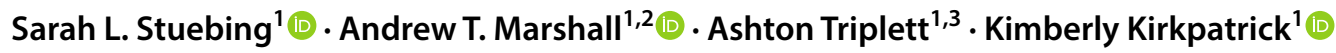

Received: 16 November 2017 / Revised: 2 August 2018 / Accepted: 7 August 2018 / Published online: 14 August 2018

(c) The Author(s) 2018

\begin{abstract}
Impulsive choice has been implicated in substance abuse, gambling, obesity, and other maladaptive behaviors. Deficits in interval timing may increase impulsive choices, and therefore, could serve as an avenue through which suboptimal impulsive choices can be moderated. Temporal interventions have successfully attenuated impulsive choices in male rats, but the efficacy of a temporal intervention has yet to be assessed in female rats. As such, this experiment examined timing and choice behavior in female rats, and evaluated the ability of a temporal intervention to mitigate impulsive choice behavior. The temporal intervention administered in this study was successful in reducing impulsive choices compared to a control group. Results of a temporal bisection task indicated that the temporal intervention increased long responses at the shorter durations. Further, results from the peak trials within the choice task combined with the progressive interval task suggest that the intervention increased sensitivity to delay and enhanced timing confidence. Overall, these results indicate that a temporal intervention can be a successful avenue for reducing impulsive choice behavior in female rats, and could contribute to the development of behavioral interventions to prevent impulsive choice and maladaptive behaviors that can be applied to both sexes.
\end{abstract}

Keywords Impulsive choice $\cdot$ Female rats $\cdot$ Interval timing $\cdot$ Temporal intervention

\section{Introduction}

Impulsive choice behavior entails placing a higher value on smaller sooner (SS) rewards than on larger later (LL) rewards, particularly when LL rewards provide greater reward earning over time (Mazur 1987). Within impulsive choice paradigms, choosing the SS can be viewed as a failure of self-control. In humans, a tendency towards making impulsive choices has been associated with several maladaptive behaviors, including substance abuse (e.g., Perry et al.

Electronic supplementary material The online version of this article (https://doi.org/10.1007/s10071-018-1208-9) contains supplementary material, which is available to authorized users.

Sarah L. Stuebing

sstuebing@ksu.edu

1 Department of Psychological Sciences, Kansas State University, 492 Bluemont Hall, Manhattan, KS 66506, USA

2 Department of Pediatrics, Keck School of Medicine, University of Southern California, and Children's Hospital Los Angeles, Los Angeles, CA 90027, USA

3 Department of Counseling and Psychological Services, State University of New York-Oswego, Oswego, NY 13126, USA
2005), gambling (e.g., Reynolds 2006), and obesity (e.g., Bruce et al. 2011; Weller et al. 2008), leading to the suggestion that impulsive choice is a trans-disease process (Bickel et al. 2012; Bickel and Mueller 2009).

Choice behavior is an individual difference variable that remains relatively constant over time, suggesting that impulsive choice may be a trait variable in humans (Jimura et al. 2011; Kirby 2009; Odum 2011) and rats (Galtress et al. 2012; Marshall et al. 2014; Peterson et al. 2015). Past research has focused on several psychological mechanisms that contribute to individual differences in impulsive choice behavior, including individual differences in interval timing and delay aversion (Baumann and Odum 2012; Kim and Zauberman 2009; Marshall et al. 2014; McClure et al. 2014). Marshall et al. (2014) found that impulsive rats demonstrated poor time discrimination relative to non-impulsive individuals (see also McClure et al. 2014) and that impulsive rats were less tolerant of longer delays. These results indicate that poor time discrimination, delay tolerance, and impulsive choice are interrelated, and suggest that mechanisms within the core timing system may be important targets for understanding the origins of impulsive choice. 
Given the established relationship between these factors, time-based interventions have been developed with the goal of moderating impulsive choice. Interventions used in humans have promoted self-control using an interval fading technique within the choice task, in which LL delays were gradually increased over time (or the SS delay gradually decreased; Binder et al. 2000; Dixon et al. 1998, 2003; Dixon and Holcomb 2000; Mazur and Logue 1978; Neef et al. 2001; Schweitzer and Sulzer-Azaroff 1995). Similar findings have been discovered in male rats. Specifically, exposure to long delays prior to choice testing promoted self-control (Stein et al. 2013, 2015) and these effects persisted over a period of 4 months (Renda and Madden 2016). In addition, Smith et al. (2015) exposed rats to fixed- or variable-interval schedules on the SS and LL levers and found increased self-control, which was accompanied by improved time discrimination. These findings were subsequently replicated in experienced, middle-aged male rats (Peterson and Kirkpatrick 2016), indicating that impulsive choice can be moderated even after previous experiences with different choice tasks. Together, these results suggest that targeting timing processes with delay-based interventions may be a fruitful enterprise for promoting self-control. However, the previous studies did not assess the efficacy of the time-based interventions in female rats, which was the primary goal of the present experiment.

Previous studies have reported sex differences in different sub-components of impulsivity and other related behaviors (see Weafer and de Wit 2014 for a comprehensive review). Koot et al. (2009b) found that female mice with relatively steep choice functions shifted to preferring the SS choice after a shorter delay than their male counterparts, indicating greater impulsivity in females than males. Female rats also demonstrated inferior sustained attention but better inhibitory control in a reaction time task when compared to male rats (Bayless et al. 2012). With these differences in mind, it is important to examine mechanisms of impulsive choice in female rats and the efficacy of time-based interventions in moderating impulsive choice. Indeed, in humans, previous research has reported sex differences in the fundamental mechanisms that comprise impulsive choice behavior (Cross et al. 2011; Eisler and Eisler 1992), such that neurocognitive interventions that are effective in males may not necessarily be as effective in females. Thus, a better understanding of these gender differences in rats may provide insight into targeted and effective treatments for men and women struggling with substance abuse, gambling, obesity, or other issues linked to impulsive choice (e.g., Perry et al. 2005; Reynolds 2006, and; Weller et al. 2008).

To our knowledge, there has been no previous attempt to assess interval timing and choice in female rats to determine whether time-based interventions may be effective in moderating impulsive choice. Accordingly, the goal of this study was to determine the effect of a fixed-interval time-based intervention on female rats' impulsive choice and timing behaviors. Additionally, the relationship between individual differences in impulsive choice behavior and timing was assessed to examine the potential role of timing processes in impulsive choice in females, thereby addressing this gap in the previous literature.

\section{Method}

\section{Animals}

Twenty-four experimentally naïve female Sprague-Dawley rats (Charles River; Kingston, NY) were used in this experiment; this strain has been used in similar research with male rats (Peterson and Kirkpatrick 2016; Smith et al. 2015). The animals arrived at the facility (Kansas State University; Manhattan, KS) at 37 days of age and began behavioral testing at 9 weeks of age. Rats were fed standard lab chow in their home cage. Food restriction began at 8 weeks of age. Using growth charts obtained from the supplier, feeding was adjusted to maintain the animals at an average of $85 \%$ of their free-feeding body weights. On testing days, feeding was also adjusted for food that each rat consumed in their experimental session, such that task performance did not affect the total amount of daily food that an individual received. However, given that animals differed in their freefeeding body weights $(\mu=168 \mathrm{~g}, \sigma=8.7 \mathrm{~g}$ ) and responded differently to food restriction, the percent body weights fluctuated during the experiment $(\mu=86.98 \%, \sigma=2.34 \%)$. The rats were provided ad libitum access to water throughout the entire experiment. They were pair-housed and maintained on a reverse 12-h light-dark cycle with all testing conducted during the dark phase of the cycle. One rat was lost due to health reasons prior to the end of the study, which resulted in the loss of the last 2 days of temporal bisection data for that animal.

\section{Apparatus}

All phases of the experiment were conducted in 24 operant chambers (Med Associates; St. Albans, VT) that were approximately $25 \times 30 \times 30 \mathrm{~cm}$. The chambers were housed within ventilated sound-attenuating isolation chambers measuring approximately $74 \times 38 \times 60 \mathrm{~cm}$. The operant chambers contained two retractable levers (ENV-122CM), two nose poke keys with cue lights (ENV-119M-1), a food receptacle (ENV-200R7), a water bottle, and a house light (ENV-215). The house light was positioned in the topcenter of the back wall. The levers were located on either side of the food receptacle at approximately one-third of the total height of the chamber. The nose poke keys with 
cue lights were located directly above each lever. A magazine pellet dispenser (ENV-203) delivered 45-mg food pellets (Bio-Serv; Flemington, NJ) into the food receptacle. A water bottle was mounted outside of the chamber directly opposite the food cup, and was accessible to the animal via a spout that ran through a hole in the lower center of the back wall. MED-PC IV controlled the experiment and recorded the time of events with a $2 \mathrm{~ms}$ resolution (Tatham and Zurn 1989).

\section{Procedure}

Rats received initial training to eat from the food magazine and press the levers and then were exposed to an impulsive choice task, followed by a temporal intervention (or control) task and then a retest of the impulsive choice task. Following the post-intervention impulsive choice task, the rats underwent a temporal bisection task and a progressive interval (PI) task in a counterbalanced order. The delays experienced within both the bisection and PI tasks were the same in all groups. The experimental tasks, as well as the sessions of each, have been provided in Table 1.

Table 1 Experimental methods

\begin{tabular}{ll}
\hline Task and phases & Sessions \\
\hline Impulsive choice, pre-intervention & \\
SS $=5 \mathrm{~s}, \mathrm{LL}=30 \mathrm{~s}$ & 10 \\
$\mathrm{SS}=10 \mathrm{~s}, \mathrm{LL}=30 \mathrm{~s}$ & 20 \\
$\mathrm{SS}=20 \mathrm{~s}, \mathrm{LL}=30 \mathrm{~s}$ & 15 \\
Temporal intervention & \\
Group FI: SS $=10 \mathrm{~s}$ & 15 \\
Group ND: FR2 $=1$ pellet with $70 \mathrm{~s}$ ITI & 15 \\
Group FI: LL $=30 \mathrm{~s}$ & 30 \\
Group ND: FR2 $=2$ pellets with $90 \mathrm{~s}$ ITI & 30 \\
Impulsive choice, post-intervention & \\
SS $=5 \mathrm{~s}, \mathrm{LL}=30 \mathrm{~s}$ & 15 \\
SS $=10 \mathrm{~s}, \mathrm{LL}=30 \mathrm{~s}$ & 15 \\
SS $=20 \mathrm{~s}, \mathrm{LL}=30 \mathrm{~s}$ & 10 \\
Temporal bisection & \\
Initial training & 10 \\
Testing 1 & 4 \\
Re-training & 2 \\
Testing 2 & 4 \\
Progressive interval & \\
PI duration $=5 \mathrm{~s}$ & 3 \\
PI duration $=10 \mathrm{~s}$ & \\
PI duration $=20 \mathrm{~s}$ & 3 \\
\hline
\end{tabular}

The length of each task and phase in sessions. Every animal experienced the same number of sessions across all tasks and phases

\section{Initial training task}

All rats were trained to eat from the food receptacle and press the levers in the operant chambers. During the magazine training session, 120 food pellets were delivered on a random time (RT) 60-s schedule. Subsequently, rats underwent 3 days of lever-press training that involved the delivery of food pellets on fixed ratio (FR) 1, random ratio (RR) 3, and RR 5 schedules of reinforcement, in which each schedule was delivered for a block of 20 reinforcers on each lever within a session.

\section{Impulsive choice task, pre-intervention}

The impulsive choice task consisted of three phases, during which rats chose between a smaller sooner (SS) reward and a larger later (LL) reward (Smith et al. 2015). Each session involved a random intermixture of free-choice, forced-choice, and peak trials. On free-choice trials, both levers were available and both delivered food on a responseinitiated fixed interval (FI) schedule. After one lever was pressed, the other lever retracted, the cue light above the pressed lever illuminated, and the FI schedule began. The first lever press following the FI delay resulted in food delivery and cue light offset. Forced-choice trials were identical to free-choice trials, but only one lever was inserted. Peak trials were similar to forced-choice trials, but trials lasted for $90 \mathrm{~s}$ and did not result in food reward. A 60-s intertrial interval (ITI) followed all trials. Each session contained 78 trials consisting of 48 free-choice, 12 SS forced-choice, 12 LL forced-choice, 3 SS peak, and 3 LL peak trials and lasted for approximately $2 \mathrm{~h}$.

The SS choice always resulted in one food pellet, and the LL choice always resulted in two food pellets. The SS delay was 5,10 , and $20 \mathrm{~s}$ in phases 1,2 , and 3 , respectively. The LL delay remained constant at $30 \mathrm{~s}$ in all phases. Each phase lasted until the rats achieved stable performance, in which choice behavior on each of the last three sessions was within $\pm 10 \%$ of the mean of those sessions. Phases 1,2, and 3 lasted for 10, 20, and 15 sessions, respectively.

\section{Temporal intervention task}

Upon completion of the pre-intervention impulsive choice task, the rats were divided into two groups-FI intervention or no-delay (ND) control-that were matched in their overall mean LL choice behavior from the pre-intervention impulsive choice task. Each trial began with the insertion of either the left or right lever and the first lever press illuminated the cue light above the lever and initiated the corresponding delay. Following a response to complete the reinforcement schedule, the cue light turned off, food was delivered, and the ITI began. Group FI was exposed to response-initiated 
FI schedules of reinforcement on both levers, such that one pellet was delivered on an FI 10-s schedule on the SS lever, and two pellets were delivered on an FI 30-s schedule of reinforcement on the LL lever. Thus, the rats received the equivalent of forced-choice trials from the choice task in the absence of any free-choice or peak trials. The ITI was $60 \mathrm{~s}$ following all trials.

Group ND was exposed to FR schedules on both levers, in which one pellet was delivered on an FR 2 schedule of reinforcement on the SS lever and two pellets were delivered on an FR 2 schedule of reinforcement on the LL lever. For Group ND, to match the rate of reinforcement on each lever experienced by Group FI, the ITI was $70 \mathrm{~s}$ on "SS" trials and $90 \mathrm{~s}$ on "LL" trials. Thus, the ND control matched the FI intervention both in terms of the minimum response requirement, the time between food deliveries, and the total food deliveries in a session.

Sessions lasted for 100 total reinforcers (100 SS or 50 LL trials). The shorter and longer FI and ND schedules were delivered in separate blocks of sessions, counterbalanced across rats. The total number of SS and LL trials was equated, such that the SS phase lasted for 15 sessions and the LL phase for 30 sessions.

\section{Impulsive choice task, post-intervention}

Following the temporal intervention task, all rats were retested in the impulsive choice task. The task was identical to the pre-intervention task except that phases 1, 2, and 3 lasted for 15,15 , and 10 sessions, respectively, to meet the stability requirement.

\section{Temporal bisection task}

The bisection task began by training the rats to discriminate a short, 4-s light signal from a long, 12-s light signal. Training trials began with the house light turning on for either a 4or 12-s duration. Once the duration elapsed, the house light turned off and both levers were inserted, allowing the rat to select the short- or long-signal lever. The levers that corresponded to SS and LL choices represented the short- and long-signal levers, respectively. Rats that chose the correct short- or long-signal lever received a one-pellet food reward and a 15-s ITI, while an incorrect choice resulted in a 5-s ITI and a correction trial, which was a repetition of the previous trial. Correction trials continued until the correct choice was made and food was delivered. Each of these training sessions consisted of 160 trials, with an equal number of presentations of each signal duration. The training sessions lasted for approximately $2 \mathrm{~h}$ and continued for 10 days, at which point the rats reached a group criterion of at least $80 \%$ correct initial choices for 2 consecutive days.
Once the training criterion was met, rats continued with test sessions consisting of a random intermixture of test trials and training trials. Training trials proceeded just as in the training phase. For each test trial, the light signal duration was randomly selected from one of the following possible durations: $4.00,5.26,6.04,6.93,7.94,9.12$, and $12.00 \mathrm{~s}$. Test trials were non-reinforced and there were no correction trials on test trials. The testing phase consisted of ten sessions: four test sessions, two re-training sessions, then another four test sessions. Each test session contained 160 training trials and 28 test trials so that each of the 7 test durations was presented four times throughout the session.

\section{PI task}

Each trial began with the insertion of the left lever. The first response after the PI delay elapsed resulted in a onepellet food delivery, lever retraction, and onset of a 5-s ITI. The delay to food delivery increased arithmetically by the PI duration for each ensuing trial until there was a pause in responding for $10 \mathrm{~min}$. The last PI duration completed prior to this pause was recorded as the breakpoint. Sessions lasted until four blocks were completed (four breakpoints were reached) or $2 \mathrm{~h}$ had elapsed. The task consisted of three phases, which employed PI durations of 5, 10, and $20 \mathrm{~s}$, and lasted for 4, 3, and 3 sessions, respectively.

\section{Data analysis}

Generalized repeated measures logistic regressions were run in MATLAB R2016a (The MathWorks; Natick, MA) on all dependent variables. Generalized repeated measures logistic regression was used for these analyses to enable the assessment of both group-level (fixed effects) and individual differences (Bolker et al. 2008; Hoffman and Rovine 2007; Young et al. 2013), as well as for the ability to include all responses as correlated observations within individuals in the analysis (Cnaan et al. 1997). The inclusion of all observations allows for a reduction in the confidence intervals surrounding the effect size estimates. Coupled with the inclusion of random effects, this results in decreased type I error rates without appreciably affecting type II error rates provided that overparameterization is avoided (Bates et al. 2015; Matuschek et al. 2017). The criterion for statistical significance was at an $\alpha$ level of 0.05 and only significant results are reported. MATLAB R2016a was also used to create all figures.

\section{Impulsive choice}

The generalized repeated measures logistic regressions were conducted with the specification of a binomial distribution and logit link function to account for the binomial distribution of the choice data-coded as 0 (SS) or 1 (LL). All 
choices during the last five sessions of each SS delay were entered into the model. The fixed effects structure included group (FI vs. ND), SS delay (5, 10, and $20 \mathrm{~s}$ ), and pre/post in a full factorial model. The best-fitting random effects structure was determined by selecting the model with the lowest Akaike's information criterion (AIC); lower AIC values indicate better fits to the data (Johnson and Omland 2004). Potential random effects included intercept, SS delay, and pre/post. If a random effect was significantly correlated with the intercept $(p<.05)$, it was removed from the random effects structure to reduce the risk of over-parameterization and loss of power (Baayen et al. 2008; Bates et al. 2015). SS delay was entered as a continuous variable and Group and pre/post were categorical variables that were effects coded $(-1,+1)$ with the control group and the pre-intervention testing as the reference levels. Scaling of the continuous variable of SS delay was adapted from Wileyto et al. (2004) and scaled relative to the 30-s LL delay (SS delay/LL delay). For the overall intercept, we determined an estimate of the predicted bias for immediacy at an SS delay of $0 \mathrm{~s}$ (Steele et al. 2017). The unstandardized regression coefficients ( $b$ values) and their 95\% confidence intervals are reported from the model and are scaled as log odds ratios.

\section{Peak timing}

Timing behavior during the impulsive choice task was assessed using rats' response rate as a function of time within both SS and LL peak trials during the last five sessions of all pre- and post-intervention phases. Response rate was computed as responses per minute in 1-s bins. Typically, peak trials induce a pattern of responding in which the rats' response rates increase until expected time of food delivery followed by a subsequent decrease in response rate (Roberts 1981). Accordingly, a modified Gaussian distribution was fit to each rat's response gradient for each phase:

$r+A \varphi(\mu, \sigma)$

in which $\varphi(\mu, \sigma)$ is a Gaussian probability density function with a mean of $\mu$ and a standard deviation of $\sigma, r$ is the operant (baseline) level of responding, and $A$ is a scaling parameter for the Gaussian function to account for individual differences in response rate. A goodness-of-fit measure (omega-squared, $\omega^{2}$ ) was calculated to determine the adequacy of the fit. The fitting of Eq. 1 was conducted using nonlinear fitting tools in MATLAB. The fits resulted in three dependent measures: (1) the time at which responding reaches its maximum rate (peak time, $\mu$ ), (2) the peak spread $(\sigma)$, and (3) the fitted response rate at the peak time (peak rate, $r$ ).

Statistical analyses of these measures involved repeated measures regression modeling as above, but with the specification of a gamma response distribution and a log link function to account for the continuous distribution of the data. These analyses involved the final five sessions of each phase. Separate models were conducted for each dependent measure from the fitting of Eq. 1. The fixed effects structure included group (FI vs. ND), SS delay, and pre/post. Group and pre/post were categorical variables as described above. The predictor of SS delay was normalized with respect to the LL delay (by dividing by $30 \mathrm{~s}$ ) to provide consistent scaling with the SS delay variable in the choice model and then centered with respect to the intermediate SS duration of $10 \mathrm{~s}$ to test effects of the intervention in the middle of the range of delays. The random effects structure was determined in the same fashion as in the choice models and included the potential random effects of intercept, pre/post, and SS delay. The unstandardized regression coefficients ( $b$ values) and their $95 \%$ confidence intervals are reported from the model and are scaled as gamma values.

\section{Temporal bisection}

Analysis of the temporal bisection task was conducted using the final 4 days of testing. Repeated measures logistic regression analyses were identical to those described for impulsive choice with the following exceptions. The predictor of signal duration was centered with respect to the geometric mean of the anchor durations (i.e., $6.93 \mathrm{~s}$ ) and normalized by dividing by the maximum signal duration (12 s). Additional tests were conducted at the anchor durations of 4 and $12 \mathrm{~s}$ by centering on those durations and normalizing the delays. The criterion was individual choices for the short- and long-signal levers (short $=0$, long $=1$ ), and the fixed effects structure included group, signal duration, and group $\times$ signal duration. Potential random effects included intercept and signal duration. The unstandardized regression coefficients ( $b$ values) and their $95 \%$ confidence intervals are reported from the model and are scaled as log odds ratios.

\section{PI task}

Repeated measures linear regression analyses were conducted on the PI task using the same general approach as with the other tasks. All sessions were included in the analysis, except for the first session of the first phase. Given relatively short session lengths, the rats did not demonstrate any breakpoints during the PI task. Therefore, two alternative measures of responding were computed. The first measure was the response rate over the whole PI duration and the second was the time of the median response within each interval. The response rate measure provided an index of delay tolerance as the PI duration increased; the median response time provided an index of temporal tracking of the increasing PI durations. Response rates were log-transformed to correct for skew and then analyzed using 
a linear regression model. A value of 0.5 was added to the response rate prior to the log transformation to account for values of zero. The unstandardized regression coefficients ( $b$ values) and their 95\% confidence intervals are reported from the model and are scaled as log response rates. The median response variable was square-root-transformed and then analyzed using a linear regression model. The PI increment variable was scaled relative to the longest PI increment by dividing by 20 . The PI duration was also relativized by scaling as a proportion of the longest PI duration that was experienced in the 20-s increment (500 s) to provide a common scaling across the three PI increments. For the median response time, any PI durations with less than two responses were removed as these trials did not contain viable bursts of responding for computing median response times. The unstandardized regression coefficients ( $b$ values) and their 95\% confidence intervals are reported from the model and are scaled as the square root of the median response times. For both response rates and median response modeling, fixed effects included group $\times$ PI increment $\times$ PI duration in a full factorial model. Potential random effects included PI increment and PI duration.

\section{Results}

\section{Impulsive choice}

Figure 1 displays the proportion of LL choices as a function of SS delay for the two groups during the pre- and postintervention tasks. The FI group made more self-controlled

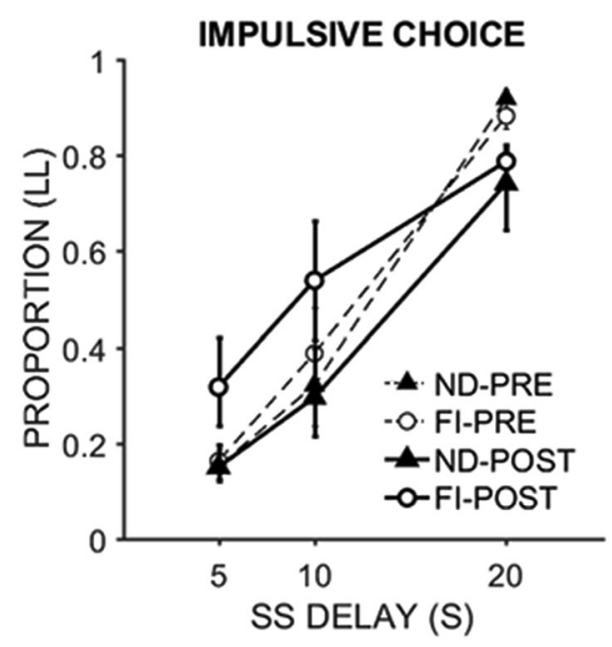

Fig. 1 The proportion of larger later (LL) choices as a function of smaller sooner (SS) delay during the pre- and post-intervention tasks in the no-delay (ND) control and fixed-interval (FI) intervention groups. The error bars are \pm the standard error of the mean based on the estimated marginal means from the model choices than their ND control counterparts across the SS delays in the post-intervention impulsive choice task, though both groups made fewer LL choices at the 20-s delay in the post-intervention task compared to pre-intervention. The regression model included random effects of intercept and pre/post. Overall, there was a significant increase in LL choices as a function of SS delay, $t(34,001)=87.59$, $p<.001, b=8.86[8.66,9.05]$. There was also a significant Group $\times$ Pre/Post interaction $t(34,001)=-2.20$, $p=.028, b=-0.37[-0.69,-0.04]$. As seen in Fig. 1, the group $\times$ Pre/Post interaction demonstrates that the two groups exhibited similar LL choice preference prior to the intervention at the 0 -s intercept $\left(b_{\mathrm{ND}}=-4.17, b_{\mathrm{FI}}=-4.23\right)$, but the ND group made significantly fewer LL choices than the FI group at the 0 -s intercept post-intervention $\left(b_{\mathrm{ND}}=-3.75, b_{\mathrm{FI}}=-2.33\right)$. The Group $\times$ Pre $/$ Post $\times \mathrm{SS}$ Delay interaction was also significant, $t(34,001)=2.67$, $p=.008, b=0.27[0.07,0.47]$, and indicated that although the two groups exhibited similar slopes pre-intervention $\left(b_{\mathrm{ND}}=10.32, b_{\mathrm{FI}}=10.62\right)$, the FI group exhibited a shallower post-intervention slope $\left(b_{\mathrm{FI}}=6.86\right)$ compared to the ND group $\left(b_{\mathrm{ND}}=7.64\right)$. This was due to the FI group's elevated LL choices at the two shortest SS delays post-intervention. Both groups also had flatter slopes in the post-intervention task due to reduced LL choices at the 20 -s delay.

\section{Peak timing}

Figure 2 displays responses per minute as a function of time in the SS and LL peak trials for the two groups during the pre- and post-intervention testing at each of the SS delays. As seen in the figure, the peak response rates generally increased during the post-intervention phase compared to the pre-intervention phase, and often were sharper. To further characterize the peak functions, the parameters from the fits of the peak functions were analyzed. Figure 3 shows the mean SS and LL peak times (a, b), peak spreads (c, d), and peak rates $(e, f)$ for each group as a function of SS delay for the SS (a, c, and e) and LL lever (b, d, and f) before (pre) and after (post) the temporal intervention. For all models analyzing SS timing, the random effects structure included intercept, and for LL timing, the random effects structure included intercept and pre/post.

\section{SS peak time}

As seen in Fig. 3a, there was the expected increase in SS peak times with increases in SS delay, $t(136)=25.95$, $p<.001, b=2.15[1.98,2.31]$, indicating that the rats learned the SS delay. There was also a significant decrease in SS peak time from the pre- to post-intervention phases, $t(136)=-3.65, p<.001, b=-0.06[-0.10,-0.03]$. This effect was largely driven by improved timing of the 5-s delay, 


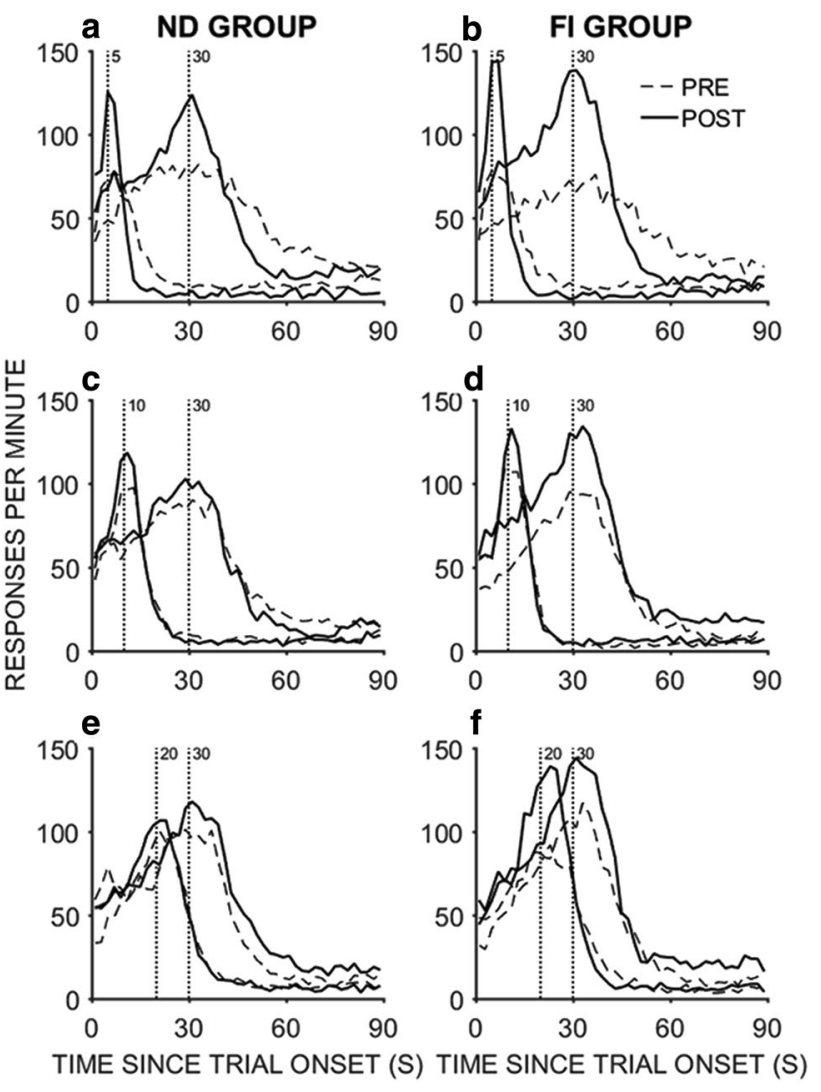

Fig. 2 Responses/min as a function of time since trial onset (s) during peak trials embedded within the pre- and post-intervention impulsive choice tasks for the no-delay (ND; a, c, e) and fixed-interval (FI; b, d, f) groups

$b_{\text {pre }}=7.8 \mathrm{~s}, b_{\text {post }}=5.8 \mathrm{~s}$, with post-intervention peak times closer to $5 \mathrm{~s}$. The slope of the SS peak time as a function of SS delay was also steeper in the post-intervention phase compared to the pre-intervention phase for both groups, Pre/ Post $\times$ SS Delay interaction, $t(136)=2.69, p=.008, b=0.22$ $[0.06,0.39]$, indicating increased sensitivity to changes in SS delay following the intervention. As this interaction was not moderated by group, these results indicate that the Pre/ Post $\times$ SS Delay interaction on SS peak times was more likely driven by additional experience with the task.

\section{SS peak spread}

As in the peak time analysis, there was no main effect of group or interactions involving group (Fig. 3c), but there was an increase in peak spread as a function of SS delay, $t(136)=18.27, p<.001, b=1.48[1.32,1.63]$, and a decrease in peak spread following the intervention phase, $t(136)=-7.95, p<.001, b=-0.14[-0.17,-0.10]$. There was also a significant Pre/Post $\times$ SS Delay interaction on SS peak spreads, $t(136)=4.36, p<.001, b=0.35[0.19,0.51]$,
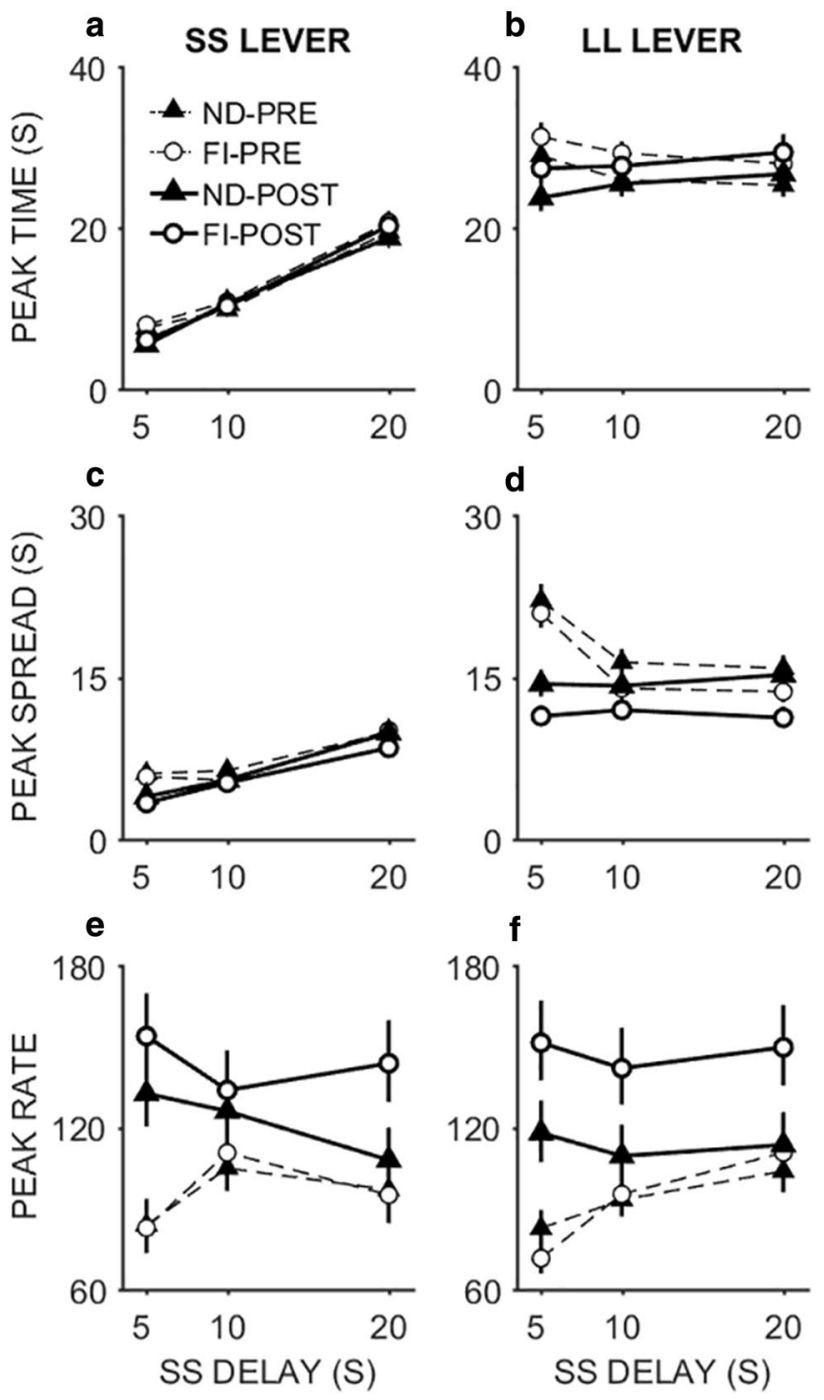

Fig. 3 Peak times (a, b), peak spreads (c, d), and peak rates (e, f) for the smaller sooner (SS) and larger later (LL) levers as a function of SS delay before and after the intervention for the no-delay (ND) and fixed-interval (FI) groups of rats. The error bars are \pm the standard error of the mean based on the estimated marginal means from the model

which was due to shallower slopes during the pre-intervention testing.

\section{SS peak rate}

SS peak rates significantly increased following the intervention (Fig. 3e), $t(136)=8.74, p<.001, b=0.16[0.12$, 0.20]. Additionally, there was a significant Pre/Post $\times \mathrm{SS}$ Delay interaction on SS peak rates, $t(136)=-2.26, p=.025$, $b=-0.20[-0.37,-0.02]$, in which there was an increase in peak rates with SS delay pre-intervention, but a decrease in peak rates with SS delay post-intervention. Overall, 
exposure to the intervention did not moderate SS peak timing measures of accuracy, precision, and rate.

\section{LL peak time}

On the LL lever, peak times were relatively constant (Fig. 3b), which is unsurprising, as the LL delay did not change throughout the experiment. There was a significant decrease in LL peak times post-intervention, $t(136)=-2.34$, $p=.021, b=-0.04[-0.08,-0.01]$, but there was no effect of group on LL peak times. Additionally, there was a significant Pre/Post $\times$ SS Delay interaction on LL peak times, $t(136)=3.60, p<.001, b=0.23[0.10,0.36]$, in that peak times decreased slightly as a function of SS delay in the pre-intervention task but increased slightly as a function of SS delay in the post-intervention task. This likely reflects learning the LL delay in the pre-intervention testing then re-adapting to the LL delay in the post-intervention testing.

\section{LL peak spread}

There was no main effect of group or interactions involving group on LL peak spreads (Fig. 3d). LL peak spreads decreased significantly with SS delay, $t(136)=-4.22$, $p<.001, b=-0.33[-0.48,-0.17]$, and post-intervention, $t(136)=-6.05, p<.001, b=-0.16[-0.21,-0.11]$, likely reflecting increased experience with the interval (i.e., more experience resulting in greater precision). Moreover, there was a significant Pre/Post $\times$ SS Delay interaction, $t(136)=4.73, p<.001, b=0.37[0.21,0.52]$, which was primarily driven by wider LL peak spreads during the preintervention exposure to the 5-s SS delay (i.e., the first phase of the experiment during which the rats were first exposed to and first learning the LL delay).

\section{LL peak rate}

LL peak rates increased significantly with SS delay (Fig. 3f), $t(136)=4.50, p<.001, b=0.29[0.16,0.42]$, and post-intervention, $t(136)=6.79, p<.001, b=0.17[0.12,0.22]$, also reflecting greater experience with the interval. Analysis also revealed a significant Pre/Post $\times$ SS Delay interaction, $t(136)=-5.05, p<.001, b=-0.32[-0.45,-0.20]$, such that the increase in LL peak rates with SS delay was steeper pre-intervention. Interestingly, while both groups exhibited similar peak rates across SS delays on the LL lever prior to the intervention, the groups diverged post-intervention. Specifically, there was a significant Group $\times$ Pre/Post interaction, $t(136)=2.96, p=.004, b=0.08[0.03,0.13]$, in which the FI group showed higher response rates around the expected time of LL reward than the ND group post-intervention $\left(b_{\mathrm{ND}}=4.68, b_{\mathrm{FI}}=4.94\right)$.

\section{Temporal bisection}

The final model included a random intercept. Figure 4 shows the proportion of long lever responses as a function of signal duration. At the geometric mean of $6.93 \mathrm{~s}$, the FI group $(0.41 \pm 0.02)$ was more likely to report the signal as long compared to the ND group $(0.38 \pm 0.02), t(2592)=2.48$, $p=.013, b=0.16[0.03,0.28]$. This is consistent with the pattern in Fig. 4 of the FI group showing increased long choices at the geometric mean relative to the ND group. In addition, the FI group was closer to indifference at the geometric mean than the ND group $\left(b_{\mathrm{FI}}=-0.20, b_{\mathrm{ND}}=-0.51\right)$, indicating that they reached their point of indifference closer to the geometric mean. Tests of the group differences at the two anchor durations revealed that the FI group produced more long responses at the 4-s duration $(\mathrm{FI}=0.21 \pm 0.02$; $\mathrm{ND}=0.08 \pm 0.01), t(2592)=2.83, p=.005, b=0.27[0.08$, $0.46]$, but there were no differences at the 12-s duration $(\mathrm{FI}=0.88 \pm 0.02, \mathrm{ND}=0.85 \pm 0.02), t(2592)=-0.37$, $p=.709, b=-0.05[-0.29,0.20]$. There also was a significant increase in long responses with increases in signal duration, $t(2592)=22.03, p<.001, b=6.03[5.49,6.56]$. There was no Group $\times$ Signal Duration interaction.

\section{PI task}

The best-fitting model for the response rates included a random intercept. There were significant effects of PI increment, $t(8025)=4.64, p<.001, b=0.26[0.15,0.37]$, PI duration, $t(8025)=-23.00, p<.001, b=-2.07[-2.25$, $-1.90]$, and Group $\times$ PI Duration $t(8025)=-3.97, p<.001$, $b=-0.36[-0.53,-0.18]$. Figure 5 shows the nature of the significant Group $\times$ PI Duration interaction. Although

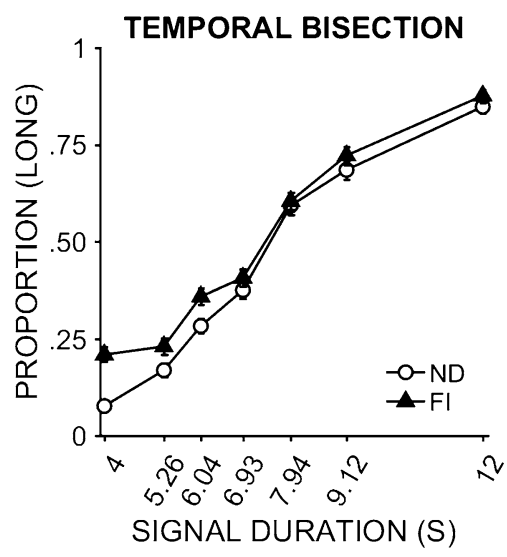

Fig. 4 Proportion of long duration responses made across each time duration experienced by the no-delay (ND) and fixed-interval (FI) groups during the bisection task. The error bars are \pm the standard error of the mean based on the estimated marginal means from the model 

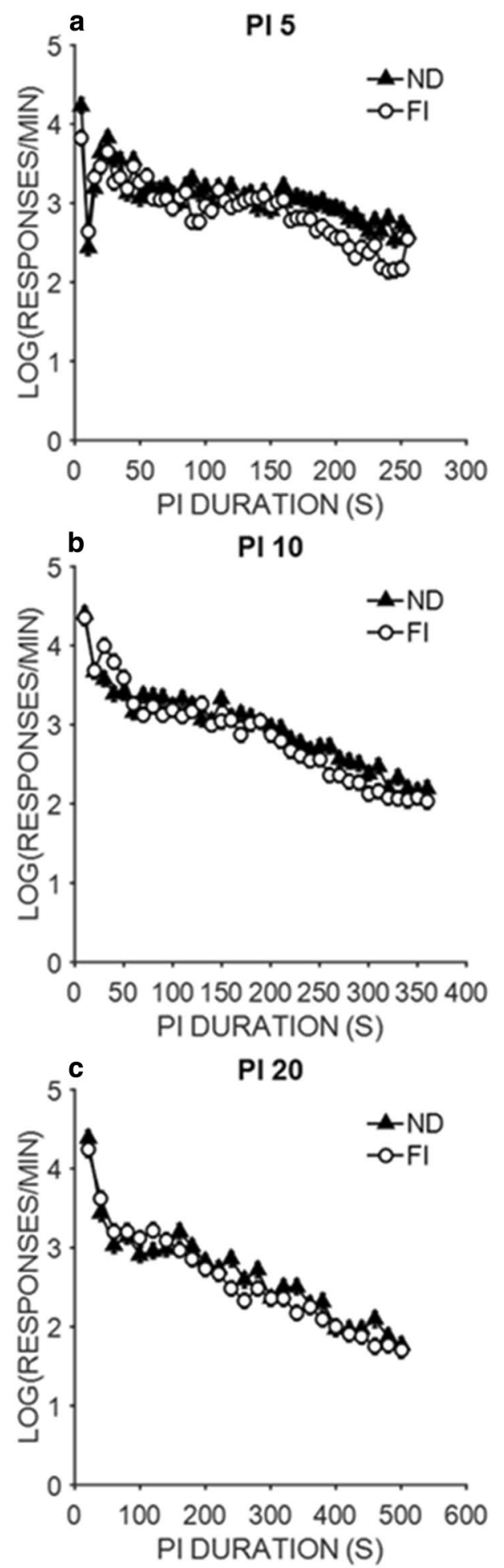

Fig. 5 Response rate (in responses/min) as a function of the PI duration (in seconds) for the no-delay (ND) and fixed-interval (FI) groups during the progressive interval (PI) task. The error bars are \pm the standard error of the mean based on the estimated marginal means from the model both groups had similar response rates during the first PI duration, the FI group decreased their rate of responding more quickly across the PI durations within each PI increment, such that the response rates of the ND and FI groups diverged as the PI duration increased.

The regression model of the median response times included a random intercept and a random slope of PI duration. The median response time results are displayed in Fig. 6. Significant effects included main effects of PI increment $t(7964)=19.77, p<.001, b=2.05$ [1.85, 2.26], and PI duration, $t(7964)=81.93, p<.001, b=18.65$ [18.20, 19.09], as well as a PI Increment $\times$ PI Duration interaction, $t(7964)=-27.40, p<.001, b=-6.21[-6.66,-5.77]$. These results indicate that both groups tracked the durations across PI increments similarly.

\section{Exploratory body weight analysis}

Given that body weight is related to impulsive choice behavior in humans (e.g., Weller et al. 2008; Rasmussen et al. 2010), an exploratory analysis was conducted on the impulsive choice data to determine if the body weights contributed to choice behavior as well as to the efficacy of the behavioral intervention. Animals were weighed at least 6 days each week, and their percentage of free-feeding body weight was used in this analysis. The final model of this analysis included a four-way interaction of Group $\times$ Pre/ Post $\times$ SS Delay $\times$ Body Weight (with all lower interactions and main effects) and random effects of intercept and pre/post. There were several significant interactions involving body weight, Group $\times$ Pre/Post $\times$ Body Weight: $t(33,993)=-8.44, p<.001, b=-0.13[-0.16,-0.10]$; Group $\times$ SS Delay $\times$ Body Weight: $t(33,993)=-3.77$, $p<.001, b=-0.10[-0.15,-0.05]$; and Pre/Post $\times \mathrm{SS}$ Delay $\times$ Body Weight: $t(33,993)=6.02, p<.001, b=0.16$ $[0.10,0.21]$. These results indicate effects of body weight on impulsive choice behavior and receptivity to the FI intervention. Other significant effects included Group $\times$ Pre/Post $\times \mathrm{SS}$ Delay: $t(33,993)=2.23, p=.025, b=0.25$ [0.03, 0.48]; SS Delay $\times$ Body Weight: $t(33,993)=3.49, p<.001, b=0.09$ [0.04, 0.14]; Group $\times$ Body Weight: $t(33,993)=5.87$, $p<.001, b=0.09$ [0.06, 0.12]; Pre/Post $\times$ SS Delay: $t(33,993)=15.63, p<.001, b=1.78$ [1.55, 2.00], Body Weight: $t(33,993)=2.82, p=.005, b=0.04[0.01,0.08] ; \mathrm{SS}$ Delay: $t(33,993)=79.79, p<.001, b=9.08$ [8.85, 9.30]; and Pre/Post: $t(33,993)=-3.22, p=.001, b=-0.56[-0.91$, $-0.22]$.

Figure 7 displays the Group $\times$ Pre/Post $\times$ Body Weight interaction, which provides the clearest insight into a possible role of body weight on impulsive choice. To aid in visualization and simplification of data patterns, the rats were grouped, for display purposes only, by their average percentages of free-feeding body weight. These groups distributed 

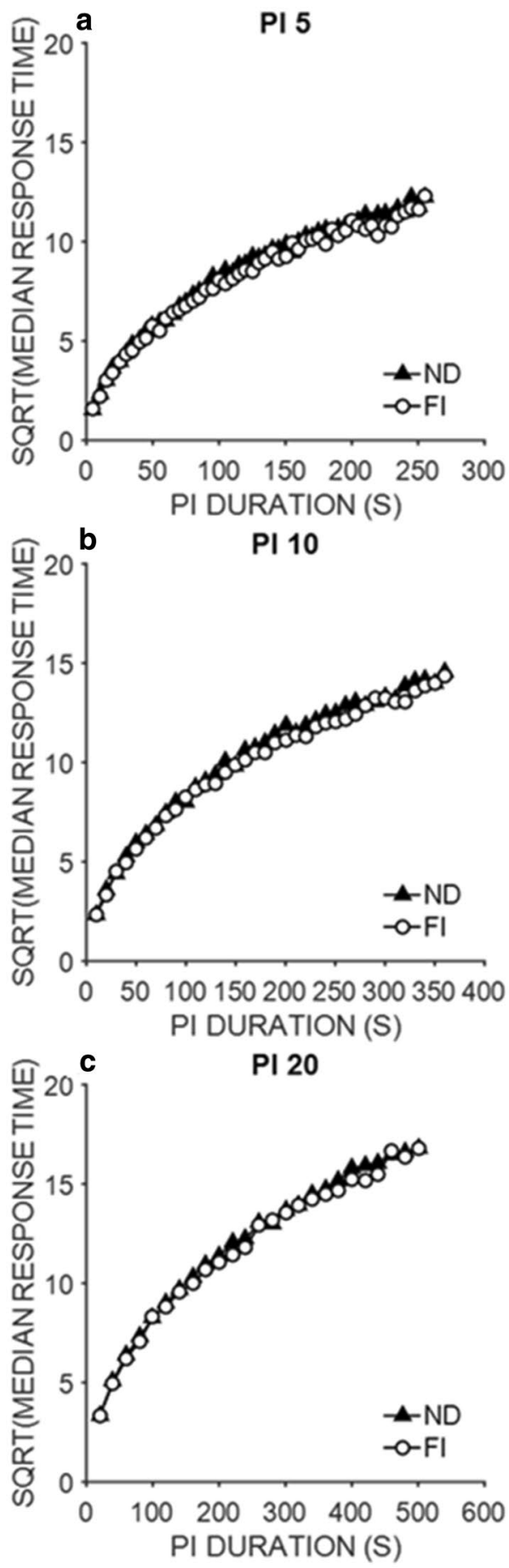

Fig. 6 Median response time (in seconds) as a function of the PI duration (in seconds) for the no-delay (ND) and fixed-interval (FI) groups during the progressive interval (PI) task. The error bars are \pm the standard error of the mean based on the estimated marginal means from the model

the number observations somewhat evenly $(N<84 \%=9591$, $\mathrm{N} 84-87 \%=10,832, N>87 \%=13,586)$ and naturally separated the individuals near the target food restriction of $85 \%$ (84-87\%), individuals lighter than the $85 \%$ target $(<84 \%)$, and individuals that remained heavier than the $85 \%$ target $(>87 \%)$. It is worth noting that rats fluctuated in weight throughout the experiment, and therefore, did not always fall into the same weight category for both pre- and postintervention. Because we wanted to measure the effect of the body weight during the current phase on choice in that phase, this issue was dealt with by including a random effect of pre/post. As seen in Fig. 7, body weight contributed to impulsive choice behavior and the effects of the FI intervention. The intervention did not promote LL choices in the lightest rats (Fig. 7a), but had a large effect in reducing impulsive choice in the middle-weight rats (Fig. 7b). Finally, the trend towards increased impulsivity in the ND control group was driven by the heaviest rats in that group. In this weight-range, the FI behavioral interventions staved off the tendency toward increased impulsivity in heavier female rats during the post-intervention choice test (Fig. 7c).

\section{Discussion}

Previous work has demonstrated that male and female rats and humans often differ in their performance on tasks measuring impulsivity (e.g., Anker et al. 2008; Bayless et al. 2012, 2015; Koot et al. 2009a; Marsh et al. 2002). Despite our knowledge that impulsive choice contributes to maladaptive behaviors, current understanding of female rodent choice behavior is sparse, as most work investigating the mechanisms of various behaviors has been conducted in male rats. However, the assumption cannot be made that male and female rats exhibit analogous choice behavior, given their biological differences. The present study provided important new findings regarding female rodent choice and timing behaviors, thus contributing to reducing a knowledge gap in the literature. The present results also provide potential insight into avenues for perturbing maladaptive impulsive choice in females.

Results from the impulsive choice task suggest that the female rats were susceptible to increased impulsivity over repeated testing. Both groups displayed flatter slopes during the post-intervention testing that was due to fewer LL choices at the 20-s SS delay (Fig. 1). Because both the FI intervention and ND control showed this pattern, the results suggest that the depressed LL responding was not due to the specific intervention condition. For example, one could argue that the ND control resulted in this increase in impulsive choices, but if the control condition were responsible then we would not have observed this same pattern in the FI intervention. Instead, it seems that the effects were due to either the passage of time, or to repeated testing on the choice task. Repeated testing could potentially increase impulsive choices for longer delays due to increased sensitivity to within-trial reward rates, which could lead to greater 

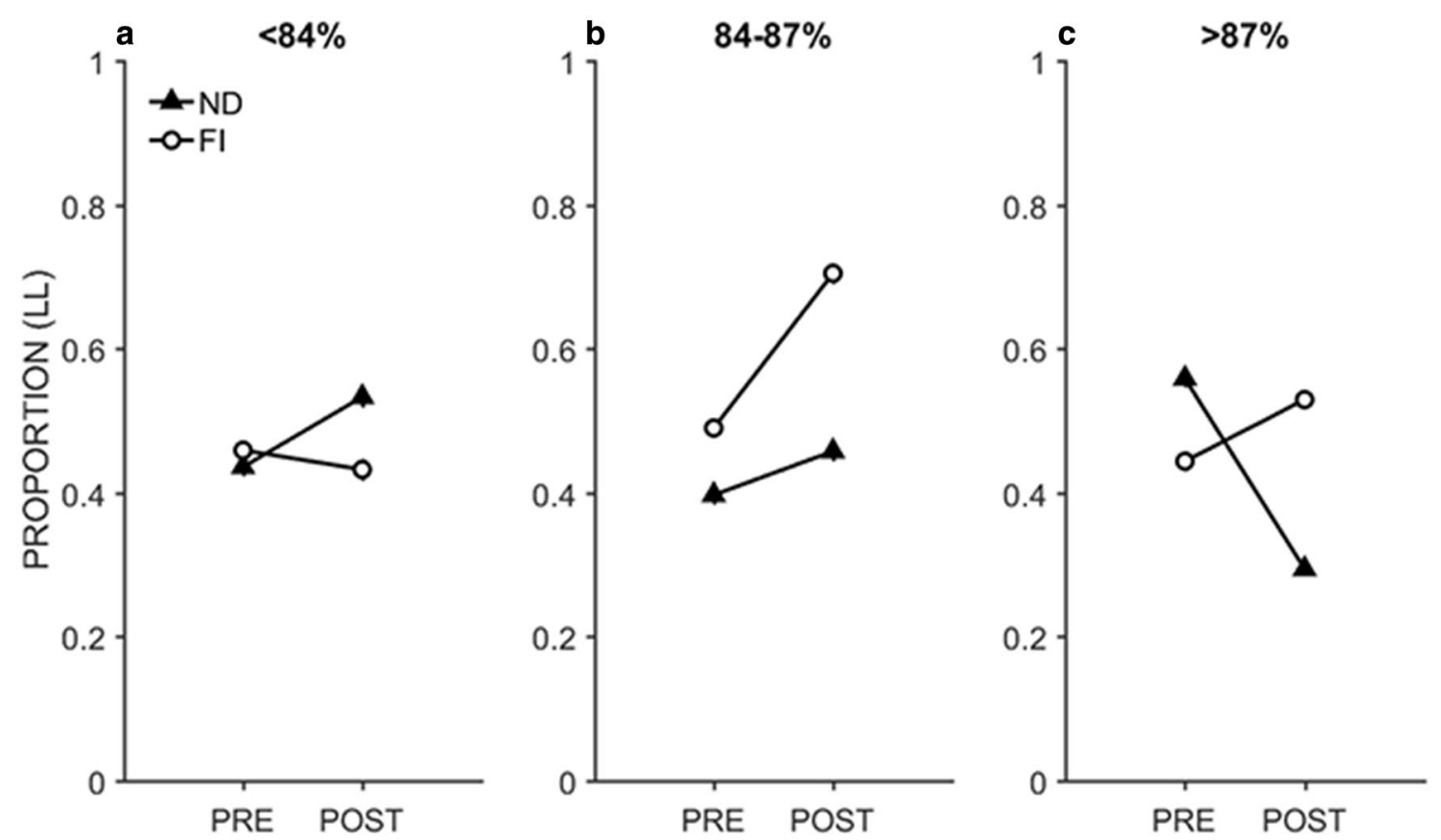

Fig. 7 Differences in choice performance in the no-delay (ND) and fixed-interval (FI) groups during the pre- and post-intervention choice task based on body weight percentage ranges. The error bars are \pm

SS preference at longer delays. Interestingly, male rats do not appear to show increased impulsive choice through repeated testing (Peterson and Kirkpatrick 2016; Smith et al. 2015), suggesting that this effect may be isolated to female rats. Further research will be needed to determine the source of these effects.

As seen in Fig. 1, the FI intervention was effective at increasing LL choices for the two shortest SS delays. Further analyses (peak trials, bisection, and PI) were conducted to determine the intervention's effects on timing behaviors. Analysis of peak times (a measure of timing accuracy) and peak spreads (a measure of timing precision) found that the rats did increase their sensitivity to the SS delay following the intervention, but this finding was not moderated by group. This suggests that timing accuracy and precision improved because of experience, but that this was not moderated by the intervention. However, the FI group did show increased LL peak response rates following the intervention, which was not seen on the SS lever. This suggests that LL timing "certainty" was improved following the intervention, meaning that the rats were more responsive at the time when food was most likely to occur.

The bisection analyses found no group differences in the slope of the function indicating that the intervention did not affect timing precision, but did improve timing accuracy. Despite an overall propensity to make short responses at the geometric mean of $6.93 \mathrm{~s}$, the FI group made significantly fewer short choices than the ND group at this delay, the standard error of the mean based on the estimated marginal means from the model

indicating more indifferent performance at the geometric mean. This suggests improved timing accuracy in the FI group as bisection typically occurs at the geometric mean in rats (e.g., Church and Deluty 1977). However, the FI group also made more long responses at the 4-s anchor duration, suggesting that the intervention induced a bias to report shorter delays as long, in comparison to the ND group. Thus, the improvement at the geometric mean may reflect a perceptual or response bias rather than increase in timing accuracy. Performance on long delays was not affected by the intervention. The bisection data may at least partially explain the increased LL choices as the subjective duration of short delays may have been increased by the intervention, which would decrease the subjective value of short delays. Overall, the bisection results differ from previous results in male rats where intervention efficacy in improving choice correlated with improvements in bisection timing precision (Peterson and Kirkpatrick 2016). However, there was more than 1 month between the end of the intervention and testing on the timing tasks. It is possible that any effect of the intervention had dissipated. Male rats do show test-retest reliability for choice that last at least 5 months (Peterson et al. 2015) and they show persistent effects of fixed interval interventions that last for at least 4-9 months (Bailey et al. 2018; Renda and Madden 2016). Although longevity and stability of choice and timing have not been previously assessed, the pre/post data here suggest poorer longevity in behavioral performance in female rats. These patterns 
highlight the potential need for a further evaluation of the effects of the intervention on impulsive choice and timing in females.

Consistent with the effects of the intervention on LL peak rates, median response rate in the PI task (Fig. 5), but not median response time (Fig. 6), was significantly altered by the intervention. Specifically, during the PI task, the FI group exhibited a steeper decline of response rates across PI durations. These results suggest improved timing certainty in the FI group, as the FI rats' response rates more accurately tracked expectations of the time of food delivery. Increased timing certainty following temporal intervention has also been observed in male rats (Smith et al. 2015), suggesting a possible common mechanism of the intervention effects between males and females. The response rate effects in females are likely not due to overall motivational differences, as all rats responded similarly at the start of the PI sessions and showed increased peak rates in the choice task. This supports the hypothesis that the intervention improved the FI group's ability to adjust response rates, whether that was in the form of more concentrated responding in peak trials (i.e., increased timing certainty) or more efficient responding across the PI sessions.

One factor that may explain the increased impulsivity in the control rats compared to their male counterparts is that female rats have been reported to show increased impulsivity when under mild food restriction (Koot et al. 2009a). Differences in sensitivity to food restriction may relate to the effect of body weight in moderating the choice behavior of both groups over time (Fig. 7). During the post-intervention impulsive choice task, the lightest animals showed no increase in LL choices, the middle-weight rats improved significantly, and the heavier animals improved only slightly. Further, the heaviest ND rats increased their impulsive choices during their post-intervention task; thus, this subgroup of rats was responsible for the overall trend towards increased impulsive choice in the ND group during the postintervention task. These results indicate that female rats' propensity toward impulsive behaviors over time may be a product of their body weights. These initial findings merit further research, as they could allow for increased success in reducing impulsive choice behavior through the application of more targeted intervention techniques that account for individuals' body weights, and could provide further insights into the mechanisms of sex differences in impulsive choice.

Though males and females were not directly compared in this work, conducting an experiment similar to previous work using male rats elucidated that sex alone inhibits exact replication of choice and timing behavior. The present findings highlight many features of female rat choice behavior, including unique results not previously found in males. As such, these results not only provide new knowledge of female rat choice behavior, but also indicate that future work involving the direct comparison of sex differences is critical to further advance the understanding of maladaptive impulsive choice behavior, as well as how to curb it. These findings also unveil some promising avenues for future work. For example, a timing intervention can successfully reduce impulsive choice behavior in female rats, though the mechanism may be due to increased timing certainty (a possible common mechanism between males and females) with some possible additional effect on timing accuracy, but in the absence of any effects on timing precision (as has been shown in males). Body weight differences may help explain why some individuals were more susceptible to impulsive choice behavior and to the intervention effects, providing a possible opportunity for the development of interventions that are specific to an individual's metabolic or energy budget needs. Ultimately, these results demonstrate that a more multi-dimensional intervention approach may more effective in mitigating impulsive choices in female rats.

Acknowledgements This research was supported by NIMH R01 Grant 085739 awarded to Kimberly Kirkpatrick and Kansas State University.

Author contributions SLS and ATM collected and analyzed data and wrote the paper. AT collected data and wrote the paper. KK conceived and designed the study and wrote the paper. The results were presented at the Society for Neuroscience and Comparative Cognition Society meetings.

\section{Compliance with ethical standards}

Conflict of interest There are no conflicts of interest to report. The manuscript has not been published previously and is not under consideration for publication elsewhere. All authors have contributed significantly to the manuscript and consent to having their names on the manuscript.

Animal welfare All applicable international, national, and/or institutional guidelines for the care and use of animals were followed. All procedures performed in studies involving animals were in accordance with the ethical standards of Kansas State University. The research was conducted under approved Institutional Animal Care and Use Protocol \#3446.

Open Access This article is distributed under the terms of the Creative Commons Attribution 4.0 International License (http://creativeco mmons.org/licenses/by/4.0/), which permits unrestricted use, distribution, and reproduction in any medium, provided you give appropriate credit to the original author(s) and the source, provide a link to the Creative Commons license, and indicate if changes were made.

\section{References}

Anker JJ, Gliddon LA, Carroll ME (2008) Impulsivity on a Go/ No-go task for intravenous cocaine or food in male and female rats selectively bred for high and low saccharin intake. Behav 
Pharmacol 19:615-629. https://doi.org/10.1097/FBP.0b013 e32830dc0ae

Baayen RH, Davidson DJ, Bates DM (2008) Mixed-effects modeling with crossed random effects for subjects and items. J Mem Lang 59:390-412. https://doi.org/10.1016/j.jml.2007.12.005

Bailey C, Peterson JR, Schnegelsiepen A, Stuebing SL, Kirkpatrick K (2018) Durability and generalizability of time-based intervention effects on impulsive choice in rats. Behav Proc 152:54-62

Bates D, Kliegl R, Vasishth S, Baayen H (2015) Parsimonious mixed models. arXiv:1506.04967 [stat.ME]

Baumann AA, Odum AL (2012) Impulsivity, risk taking, and timing. Behav Process 90:408-414. https://doi.org/10.1016/j.bepro c. 2012.04 .005

Bayless DW, Darling JS, Stout WJ, Daniel JM (2012) Sex differences in attentional processes in adult rats as measured by performance on the 5-choice serial reaction time task. Behav Brain Res 235:4854. https://doi.org/10.1016/j.bbr.2012.07.028

Bayless DW, Perez MC, Daniel JM (2015) Comparison of the validity of the use of the spontaneously hypertensive rat as a model of attention deficit hyperactivity disorder in males and females. Behav Brain Res 286:85-92. https://doi.org/10.1016/j. bbr.2015.02.029

Bickel WK, Mueller ET (2009) Toward the study of trans-disease processes: a novel approach with special reference to the study of comorbidity. J Dual Diagn 5:131-138. https://doi.org/10.1080/15504 260902869147

Bickel WK, Jarmolowicz DP, Mueller ET, Koffarnus MN, Gatchalian KM (2012) Excessive discounting of delayed reinforcers as a trans-disease process contributing to addiction and other diseaserelated vulnerabilities. Emerg Evid Pharmacol Ther 134:287-297. https://doi.org/10.1016/j.pharmthera.2012.02.004

Binder LM, Dixon MR, Ghezzi PM (2000) A procedure to teach selfcontrol to children with attention deficit hyperactivity disorder. J Appl Behav Sci 33:233-237

Bolker BM, Brooks ME, Clark CJ, Geange SW, Poulsen JR, Stevens MHH, White J-SS (2008) Generalized linear mixed models: a practical guide for ecology and evolution. Trends Ecol Evol 24:127-135. https://doi.org/10.1016/j.tree.2008.10.008

Bruce AS, Black WR, Bruce JM, Daldalian M, Martin LE, Davis AM (2011) Ability to delay gratification and BMI in preadolescence. Obesity (Silver Spring) 19:1101-1102. https://doi.org/10.1038/ oby. 2010.297

Church RM, Deluty MZ (1977) Bisection of temporal intervals. J Exp Psychol Anim Behav Process 3:216-228. https://doi. org/10.1037/0097-7403.3.3.216

Cnaan A, Laird N, Slasor P (1997) Tutorial in biostatistics: using the general linear mixed model to analyse unbalanced repeated measures and longitudinal data. Stat Med 16:80

Cross CP, Copping LT, Campbell A (2011) Sex differences in impulsivity: a meta-analysis. Psychol Bull 137:97-130. https://doi. org/10.1037/a0021591

Dixon MR, Holcomb S (2000) Teaching self-control to small groups of dually diagnosed adults. J Appl Behav Anal 33:611-614

Dixon MR, Hayes LJ, Binder LM, Manthey S, Sigman C, Zdanowski DM (1998) Using a self-control training procedure to increase appropriate behavior. J Appl Behav Anal 31:203-210

Dixon MR, Rehfeldt RA, Randich L (2003) Enhancing tolerance to delayed reinforcers: the role of intervening activities. J Appl Behav Anal 36:263-266

Eisler H, Eisler AD (1992) Time perception: effects of sex and sound intensity on scales of subjective duration. Scand J Psychol 33:339-358. https://doi.org/10.1111/j.1467-9450.1992.tb00923.x

Galtress T, Garcia A, Kirkpatrick K (2012) Individual differences in impulsive choice and timing in rats. J Exp Anal Behav 98:65-87. https://doi.org/10.1901/jeab.2012.98-65
Hoffman L, Rovine MJ (2007) Multilevel models for the experimental psychology: foundations and illustrative examples. Behav Res Methods 39:101-117. https://doi.org/10.3758/BF03192848

Jimura K, Myerson J, Hilgard J, Keighley J, Braver TS, Green L (2011) Domain independence and stability in young and older adults' discounting of delayed rewards. Behav Process 87:253-259. https ://doi.org/10.1016/j.beproc.2011.04.006

Johnson JB, Omland KS (2004) Model selection in ecology and evolution. Trends Ecol Evol 19:101-108. https://doi.org/10.1016/j. tree.2003.10.013

Kim BK, Zauberman G (2009) Perception of anticipatory time in temporal discounting. J Neurosci Psychol Econ 2:91-101

Kirby KN (2009) One-year temporal stability of delay-discount rates. Psychon Bull Rev 16:457-462. https://doi.org/10.3758/ PBR.16.3.457

Koot S, Adriani W, Saso L, van den Bos R, Laviola G (2009a) Home cage testing of delay discounting in rats. Behav Res Methods 41(4):1169-1176:1169-1176

Koot S, van den Bos R, Adriani W, Laviola G (2009b) Gender differences in delay-discounting under mild food restriction. Behav Brain Res 200:134-143. https://doi.org/10.1016/j.bbr.2009.01.006

Marsh D, Dougherty DM, Mathias F, Moeller G, Hicks LR (2002) Comparisons of women with high and low trait impulsivity using behavioral models of response-disinhibition and reward-choice. Personal Individ Differ 33:1291-1310

Marshall AT, Smith AP, Kirkpatrick K (2014) Mechanisms of impulsive choice: I. Individual differences in interval timing and reward processing. J Exp Anal Behav 102:86-101. https://doi. org/10.1002/jeab.88

Matuschek H, Kliegl R, Vasishth S, Baayen H, Bates D (2017) Balancing type I error and power in linear mixed models. J Mem Lang 94:305-315. https://doi.org/10.1016/j.jml.2017.01.001

Mazur JE (1987) An adjusting procedure for studying delayed reinforcement. In: Commons ML, Mazur JE, Nevin JA, Rachlin H (eds) Quantitative analyses of behavior. The effect of delay and of intervening events on reinforcer value, vol 5. Erlbaum, Hillsdale, pp 55-73

Mazur JE, Logue AW (1978) Choice in a "self-control" paradigm: effects of a fading procedure. J Exp Anal Behav 30:11-17

McClure J, Podos J, Richardson HN (2014) Isolating the delay component of impulsive choice in adolescent rats. Front Integr Neurosci 8:1-9. https://doi.org/10.3389/fnint.2014.00003

Neef NA, Bicard DF, Endo S (2001) Assessment of impulsivity and the development of self-control in students with attention deficit hyperactivity disorder. J Appl Behav Anal 34:397-408

Odum AL (2011) Delay discounting: Trait variable? Behav Process $87: 1-9$

Perry JL, Larson EB, German JP, Madden GJ, Carroll ME (2005) Impulsivity (delay discounting) as a predictor of acquisition of IV cocaine self-administration in female rats. Psychopharmacology 178:193-201. https://doi.org/10.1007/s00213-004-1994-4

Peterson JR, Kirkpatrick K (2016) The effects of a time-based intervention on experienced middle-aged rats. Behav Process 133:44-51. https://doi.org/10.1016/j.beproc.2016.11.002

Peterson JR, Hill CC, Kirkpatrick K (2015) Measurement of impulsive choice in rats: same- and alternate-form test-retest reliability and temporal tracking. J Exp Anal Behav 103:166-179. https://doi. org/10.1002/jeab.124

Rasmussen EB, Lawyer SR, Reilly W (2010) Percent body fat is related to delay and probability discounting for in humans. Behav Process 83:23-30

Renda CR, Madden GJ (2016) Impulsive choice and pre-exposure to delays: III. Four-month test-retest outcomes in male wistar rats. Behav Process 126:108-112. https://doi.org/10.1016/j.bepro c.2016.03.014 
Reynolds B (2006) A review of delay-discounting research with humans: relations to drug use and gambling. Behav Pharmacol 17:651-667

Roberts S (1981) Isolation of an internal clock. J Exp Psychol Anim Behav Process 7:242-268. https://doi. org/10.1037/0097-7403.7.3.242

Schweitzer JB, Sulzer-Azaroff B (1995) Self-control in boys with attention deficit hyperactivity disorder: effects of added stimulation and time. J Child Psychol Psychiatry 36:671-686

Smith AP, Marshall AT, Kirkpatrick K (2015) Mechanisms of impulsive choice: II. Time-based interventions to improve self-control. Behav Process 112:29-42. https://doi.org/10.1016/j.bepro c. 2014.10 .010

Steele CC, Pirkle JRA, Kirkpatrick K (2017) Diet-induced impulsivity: effects of a high-fat and a high-sugar diet on impulsive choice in rats. PLOS One 12:e0180510. https://doi.org/10.1371/journ al.pone. 0180510

Stein JS, Johnson PS, Renda CR, Smits RR, Liston KJ, Shahan TA, Madden GJ (2013) Early and prolonged exposure to reward delay: effects on impulsive choice and alcohol self-administration in male rats. Exp Clin Psychopharmacol 21:172-180
Stein JS, Renda CR, Hinnenkamp JE, Madden GJ (2015) Impulsive choice, alcohol consumption, and pre-exposure to delayed rewards: II. Potential mechanisms. J Exp Anal Behav 103:33-49. https://doi.org/10.1002/jeab.116

Tatham TA, Zurn KR (1989) The MED-PC experimental apparatus programming system. Behav Res Methods Instrum Comput 21:294-302. https://doi.org/10.3758/BF03205598

Weafer J, de Wit H (2014) Sex differences in impulsive action and impulsive choice. Addict Behav 39:1573-1579

Weller RE, Cook EW, Avsar KB, Cox JE (2008) Obese women show greater delay discounting than healthy-weight women. Appetite 51:563-569. https://doi.org/10.1016/j.appet.2008.04.010

Wileyto EP, Audrain-Mcgovern J, Epstein LH, Lerman C (2004) Using logistic regression to estimate delay-discounting functions. Behav Res Methods Instrum Comput 36:41-51. https://doi.org/10.3758/ bf03195548

Young ME, Webb TL, Sutherland SC, Jacobs EA (2013) Magnitude effects for experienced rewards at short delays in the escalating interest task. Psychon Bull Rev. https://doi.org/10.3758/s1342 3-012-0350-7 